\title{
Optimal design techniques for kinematic couplings
}

\author{
Layton C. Hale ${ }^{\mathrm{a}, *}$, Alexander H. Slocum ${ }^{\mathrm{b}}$ \\ ${ }^{a}$ Lawrence Livermore National Laboratory, Livermore, CA, USA \\ ${ }^{\mathrm{b}}$ Department of Mechanical Engineering, Massachusetts Institute of Technology, Cambridge, MA, USA
}

Received 7 March 2000; received in revised form 18 September 2000; accepted 5 October 2000

\begin{abstract}
Kinematic couplings are well known to the precision engineering community as simple devices that provide rigid, repeatable connection between two objects through usually six local contact areas. They serve many applications that require 1) separation and repeatable engagement, and/or 2) minimum influence that an imprecise or unstable foundation has on the stability of a precision component. Typically, the coupling design process starts by arranging or adapting one of two classic configurations, the three-vee coupling or the tetrahedronvee-flat coupling, to suit the geometry of the application. It is often sufficient to analyze only the contact stresses and perhaps the coupling stiffness when the configuration remains fairly conventional (i.e., planar) and the application is not particularly demanding. Otherwise, effort spent optimizing the configuration through additional analysis and/or testing is well worthwhile. This paper proposes several optimization criteria and presents analysis techniques for optimizing kinematic coupling designs. The general modeling approach uses [6 $\times 6$ ] transformation matrices to reflect contact stiffness matrices to a common coordinate system where they are added together as a parallel combination, for example. This method has wider applications particularly for flexure systems, which will be the subject of a future article. In addition, the reader may find the kinematic coupling designs presented in this paper useful for future applications. (C) 2001 Elsevier Science Inc. All rights reserved.
\end{abstract}

Keywords: Kinematic coupling; Optimization; Computer-aided design; Friction; Repeatability; Stiffness matrix; Transformation matrix; Touch-trigger probe; National Ignition Facility; NIF

\section{Introduction}

The origin of the kinematic coupling dates back at least to 1,876 when James Clerk Maxwell described the three-vee coupling as a method to establish a definite position [1,2]. He also described the tetrahedron-vee-flat coupling in use by William Thomson, now widely known as Lord Kelvin and hence the term Kelvin clamp. Evans found evidence that both Maxwell and Thompson learned kinematics from Professor Robert Willis, but apparently Willis' own publications do not support him as the innovator [2]. In particular, Thompson mentions being taught the geometrical (or kinematic) method by Willis some thirty years earlier, approximately 1,849 [3,2]. Regardless of the origin, these two classic configurations, shown in Fig. 1 provided the basis for most kinematic couplings developed since that time.

Kinematic couplings have traditionally been used in in-

$$
2419 .
$$

* Corresponding author. Tel.: +1-925-422-6823; fax: +1-925-423-

E-mail address: hale6@1lnl.gov (L.C. Hale). strument design where the loads typically are relatively light and static. Kinematic couplings having well engineered contact areas and/or advanced ceramic materials are quite robust and suitable for demanding applications requiring high stiffness and load capacity $[4,5,6]$. This paper does not present specific guidance regarding Hertz analysis or material selection as considerable information already exists in the literature [7]. Instead, it emphasizes the process of determining the best configuration for a given set of objectives and design constraints. In this regard, Maxwell contributed a very elegant and general guideline. Each constraint should be aligned to the local direction of motion allowed by the five other constraints, assuming that they remain engaged and are free to slide $[1,8,9]$. Intuitively this is a good idea because each constraint would contribute its full stiffness and carry the minimum load. For example, this guideline suggests that the faces of a vee should form a right angle. Another published guideline, specifically for planar three-vee couplings, turns out to be completely consistent with Maxwell. As Fig. 2 shows, the axis of each vee should bisect the angle formed between it and the other two vees 

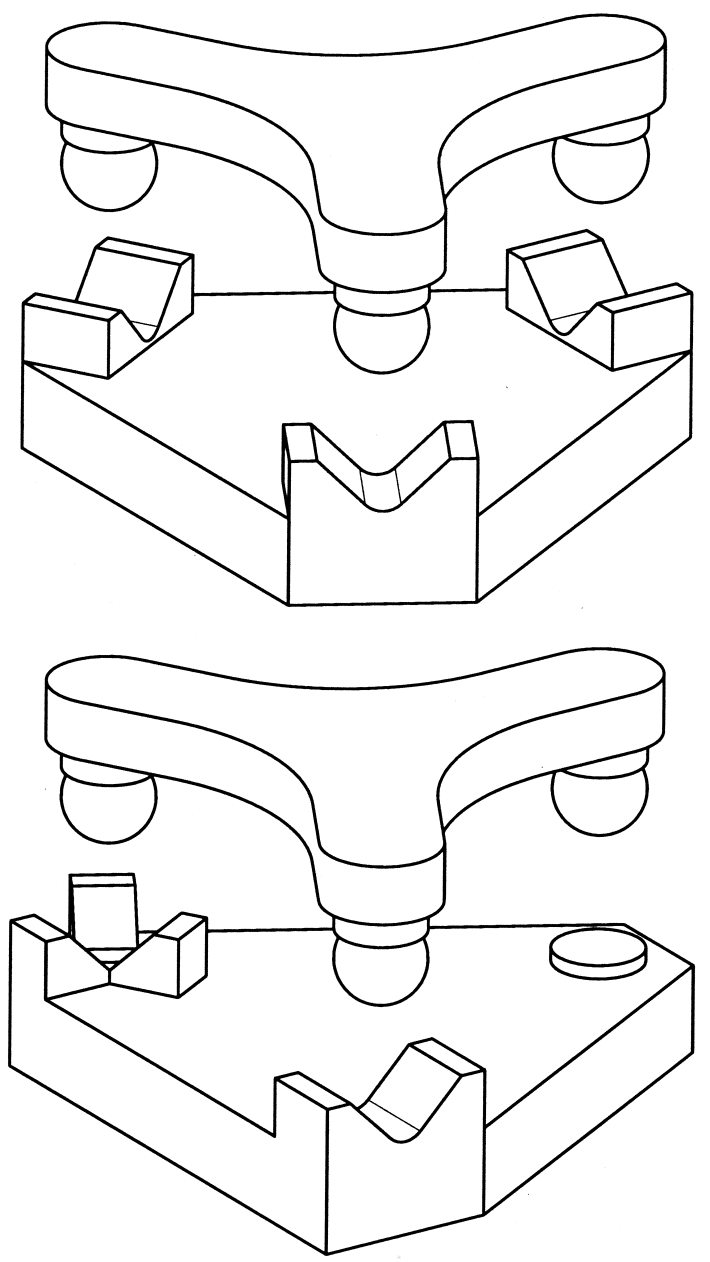

(a)

(b)

Fig. 1. In (a), the three-vee coupling has six constraints arranged in three pairs. In (b), the tetrahedron-vee-flat coupling has six constraints arranged in a 3-2-1 configuration.

$[8,9]$. The fact that this axis also intersects the instantaneous center of rotation formed by the other two vees means that rotation about this center would not produce translation along the vee. ${ }^{1}$ Maxwell's criterion is satisfied for Fig. 2 if the faces of each vee also form a right angle.

Mathematically, the system matrix describes the geometry and behavior of the coupling, both as an equilibrium condition between a vector of contact forces and the applied force-moment vector, and as a compatibility condition between a differential translation-rotation vector and a vector of contact deflections. The condition number of the system matrix has been postulated to indicate the quality of the configuration [10]. A very large condition number means that the matrix is near singular or ill conditioned. However, since the condition number also depends on the size of the coupling and the units involved, the value cannot be used in an absolute sense to evaluate the design. In contrast, the approach taken in this paper builds mathematics around some meaningful physical measure. For example, friction present in the contacting surfaces acts to impede the coupling as it slides to a fully engaged or centered position.

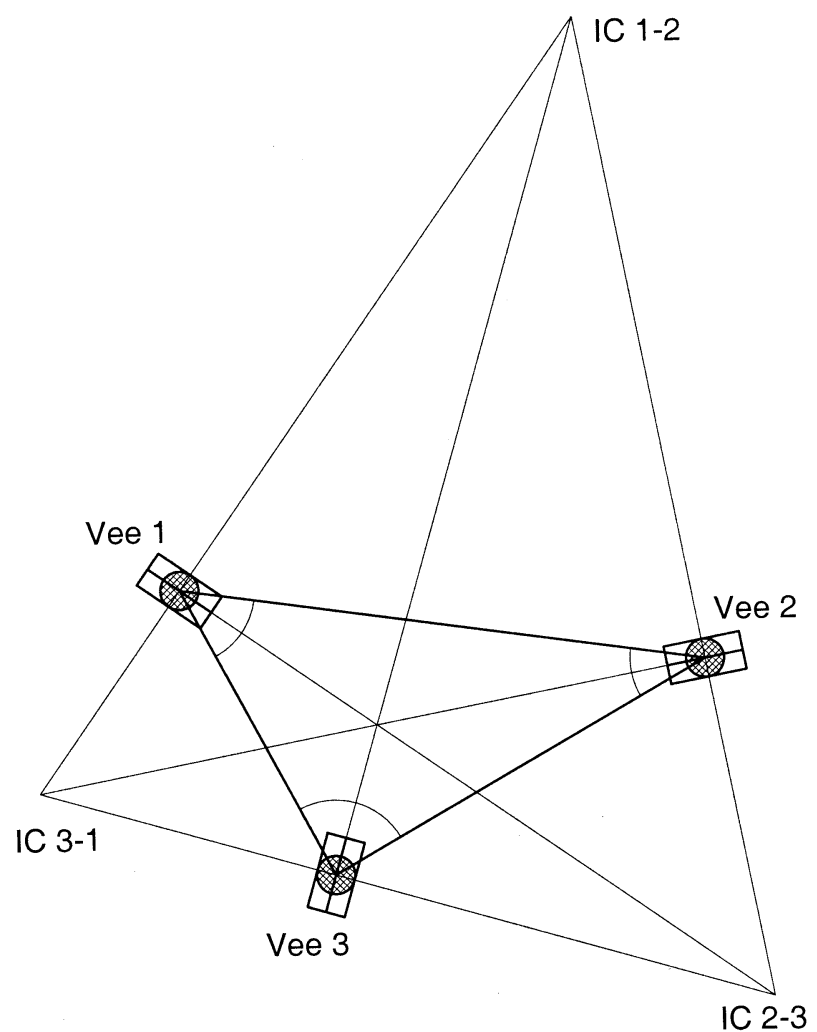

Fig. 2. This plan view of a three-vee coupling was created by arbitrarily placing three balls then orienting the vees to bisect the angles between balls. Lines projected perpendicular to any two vees intersect at instantaneous centers (IC). It so happens that the vees oriented in this manner always intersect the IC's as shown.

Thus, a coupling designed to maximize centering ability, as measured by the coefficient of friction that just impedes sliding, optimizes a meaningful physical measure. In similar fashion, a clever graphical approach to the centering problem is described in an excellent book on exact constraint design [11]. ${ }^{2}$

Friction also negatively affects the repeatability of a kinematic coupling by trapping uncertain tangential forces (in magnitude and direction) at the contacting surfaces. One approach to this problem adds tangential compliance at the contacting surfaces, for example, with flexure cuts [12]. ${ }^{3}$ The extra tangential compliance minimizes frictional hysteresis resulting from applied forces or temperature variations. A fairly common suggestion for relieving residual friction forces is the temporary application of vibrational energy, but this does not address frictional hysteresis. One approach explored in this paper optimizes the coupling configuration for repeatability.

\subsection{Organization}

The body of this paper begins by explaining the basic modeling approach using $[6 \times 6]$ transformation matrices. This introduces the reader to the technique and the reasons 
why it is preferred over a more direct approach such as that used by Schmiechen [10]. The details required for implementation are postponed to the Appendix. Then four optimization criteria are developed to satisfy a range of applications. These appear in a generally increasing order of complexity, the next one extending an idea beyond the previous ones: 1) Maxwell's criterion, 2) maximizing the modal frequency, 3) minimizing frictional nonrepeatability, and 4) maximizing the limiting coefficient of friction. The last criterion was introduced at the 1998 ASPE Conference and later documented in full [13,14]. A fifth criterion more specific for the design of touch-trigger probes will be the subject of a future article. Finally the application that motivated this study, kinematic mounts for replaceable optics assemblies in the National Ignition Facility (NIF), serves to demonstrate a graphical approach to multi-variable optimization.

\section{Modeling approach}

It is sufficient, for the purposes of optimizing the configuration or determining the approximate stiffness, to represent the kinematic coupling as a system of springs, typically as a parallel combination of six springs. The basic assumption is that the equivalent spring for the combination represents useful information, for example, the equivalent stiffness matrix. If desired, this information is easily propagated back to individual springs, for example, to obtain local forces and moments. Additional springs may be included to represent additional sources of compliance such as added flexures and the coupling body. Usually each spring is linear or is linearized about a nominal load so it can be represented as a stiffness or compliance matrix, although the complete nonlinear vector function may be retained if desired.

The key formalism in this approach is the six-dimensional vector used to succinctly represent three linear degrees of freedom and three angular degrees of freedom. This formalism is common in the literature for robot manipulators [15]. For example, the vector may be a force-moment vector or a differential translation-rotation vector, which describes the load or deflection, respectively, of a six-dimensional spring. The linear relationship between the load and deflection is represented by the $[6 \times 6]$ stiffness matrix or its inverse the compliance matrix. It is convenient to express $[6 \times 6]$ matrices as blocks of $[3 \times 3]$ matrices as demonstrated with the stiffness matrices in Eqs. (1) and (2). In practice it is usually easier to construct the compliance matrix in Eq. (3), then invert the whole matrix to obtain the stiffness matrix. For example, each column of the compliance matrix contains the translation-rotation vector obtained by the independent application of the unit force or moment component corresponding to that column.

$$
\begin{aligned}
& \mathbf{f}=\left[\begin{array}{l}
f_{x} \\
f_{y} \\
f_{z}
\end{array}\right]=\left[\begin{array}{lll}
k_{x x} & k_{x y} & k_{x z} \\
k_{x y} & k_{y y} & k_{y z} \\
k_{x z} & k_{y z} & k_{z z}
\end{array}\right] \cdot\left[\begin{array}{l}
d \delta_{x} \\
d \delta_{y} \\
d \delta_{z}
\end{array}\right]=\mathbf{K}_{\mathbf{f} / \boldsymbol{\delta}} \cdot \mathrm{d} \boldsymbol{\delta} \\
& {\left[\begin{array}{c}
\mathbf{f} \\
\hdashline- \\
\mathbf{m}
\end{array}\right]=\left[\begin{array}{l}
\mathbf{K}_{\mathbf{f} / \boldsymbol{\delta}} \\
\hdashline \mathbf{K}_{\mathbf{f} / \boldsymbol{\theta}} \\
\hdashline \mathbf{K}_{\mathbf{m} / \boldsymbol{\delta}}: \mathbf{K}_{\mathbf{m} / \boldsymbol{\theta}}
\end{array}\right] \cdot\left[\begin{array}{c}
d \boldsymbol{\delta} \\
-- \\
\mathrm{d} \boldsymbol{\theta}
\end{array}\right] \quad \mathbf{K}_{\mathbf{m} / \boldsymbol{\delta}}=\mathbf{K}_{\mathbf{f} / \boldsymbol{\theta}}{ }^{\mathrm{T}}} \\
& {\left[\begin{array}{c}
d \boldsymbol{\delta} \\
-- \\
\mathrm{d} \boldsymbol{\theta}
\end{array}\right]=\left[\begin{array}{l:l}
\mathbf{C}_{\boldsymbol{\delta} / \mathbf{f}} & \mathbf{C}_{\boldsymbol{\delta} / \mathbf{m}} \\
\hdashline \mathbf{C}_{\boldsymbol{\theta} / \mathbf{f}} & \mathbf{C}_{\boldsymbol{\theta} / \mathbf{m}}
\end{array}\right] \cdot\left[\begin{array}{c}
\mathbf{f} \\
-- \\
\mathbf{m}
\end{array}\right] \quad \mathbf{C}_{\boldsymbol{\theta} / \mathbf{f}}=\mathbf{C}_{\boldsymbol{\delta} / \mathbf{m}}{ }^{\mathrm{T}}}
\end{aligned}
$$

Any spring has a principal coordinate system (CS) where the off-diagonal terms of the stiffness or compliance matrix are zero. In defining either matrix, it is most convenient to use the principal CS or perhaps another CS where the spring is largely symmetric. The Hertz contact problem is defined in such a CS where the origin is at the center of the contact and the $z$-axis is normal to the contacting surfaces. If the contact is assumed to be friction free or in a state of sliding, then only the $k_{z z}$ element is nonzero. If instead no slip occurs at the contact, then $k_{x x}$ and $k_{y y}$ will have up to $83 \%$ the value of $k_{z z}$, depending on the lateral load and other factors [7]. In either case, the $[6 \times 6]$ stiffness matrix will become full, in general, when reflected to a different CS, perhaps one at the center of the kinematic coupling. Once all the stiffness matrices for the six contact surfaces are reflected to the same CS, then it is a simple matter to add them together to get the stiffness matrix for the kinematic coupling in that common CS. The $[6 \times 6]$ transformation matrix makes the process of reflecting a stiffness or compliance matrix to a different CS straightforward.

\subsection{Transformation-matrix basics}

The $[3 \times 3]$ rotation matrix and the $[4 \times 4]$ homogeneous transformation matrix (HTM) are well known tools that many readers will find familiar. Since these are important in understanding the lesser known $[6 \times 6]$ transformation matrix used here, brief descriptions are provided for those that require review. The rotation matrix describes in its three columns the angular orientation of the $x-y-z$ axes, respectively, of a new CS with respect to the base CS. Multiplying the rotation matrix by a three-dimensional vector expressed in the new CS transforms its representation to the base CS as if they share a common origin. ${ }^{4}$ The rotation matrix is orthonormal, thus placing six constraints on its nine elements: three orthogonality conditions and three unitlength conditions (the unit vectors of an orthogonal CS). This leaves three independent parameters that are sufficient to represent any given angular orientation, usually as a series of three angular dimensions taken in a specific order. The rotation matrix is basic to other more specialized transformation matrices such as the HTM. Applicable only to position vectors, the HTM contains the rotation matrix and 


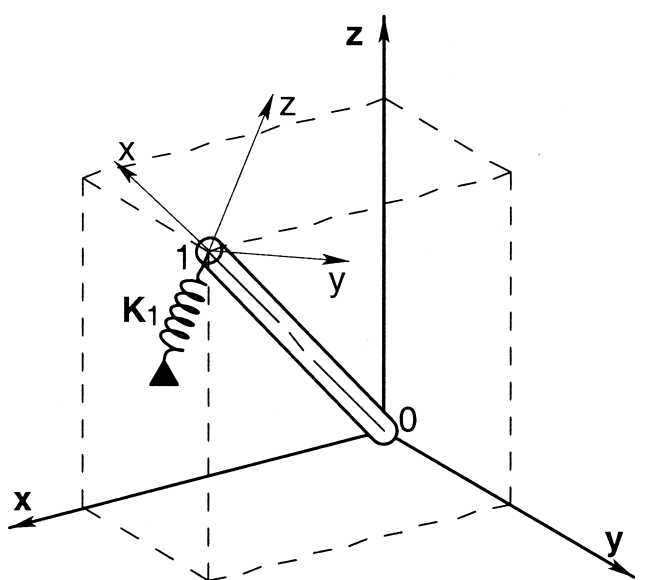

Fig. 3. The stiffness matrix $\mathbf{K}_{1}$ of the spring is expressed in terms of the local $\mathrm{CS}_{1}$. The goal is to express the stiffness matrix in terms of the base $\mathrm{CS}_{0}$. A collection of springs once represented in the same CS can be added together in series or parallel combinations.

accounts for a translation between CS's, changing the representation of a position in the new CS to the same position in the base CS. Likewise, the $[6 \times 6]$ transformation matrix accounts for both rotation and translation between two CS's but is applicable only to six-dimensional vectors such as those described previously. ${ }^{5}$

A sequence of transformations through a chain of CS's is conveniently represented as a sequence of transformation matrices multiplied together as shown in Eq. (4). The fractional subscripts are useful to keep the proper order and sense of the matrices. This sequence may be considered from two different points of view. If each transformation is described with respect to a body CS that changes through the sequence, then the sequence progresses from left to right in Eq. (4) from the base $\mathrm{CS}_{0}$ to the newest $\mathrm{CS}_{\mathrm{n}}$. If instead each transformation is described with respect to the base $\mathrm{CS}_{0}$, then the sequence progresses from right to left in Eq. (4). These two points of views have important practical value when dealing with sequential transformations and may be summarized with two simple rules [15]:

Rule 1: post multiply to transform in body coordinates

Rule 2: pre multiply to transform in base coordinates

$$
\begin{aligned}
\mathbf{v}_{0} & =\mathbf{T}_{0 / 1} \cdot \mathbf{T}_{1 / 2} \cdots \mathbf{T}_{n-1 / n} \cdot \mathbf{v}_{n} \\
& =\mathbf{T}_{0 / n} \cdot \mathbf{v}_{n}
\end{aligned}
$$

\subsection{Equations of compatibility and equilibrium}

The $[6 \times 6]$ transformation matrix is derived from compatibility and equilibrium equations, which express the same geometric relationship, compatibility as a consistent relationship among displacements and equilibrium as a balance of forces. It is instructive to work through the example in Fig. 3, which shows one spring in what could be a parallel and/or series combination with many other springs. One end of the spring is grounded and the other is connected to a rigid link that extends to the base $\mathrm{CS}_{0}$. Movement of the link at $\mathrm{CS}_{0}$ causes the spring to deflect relative to $\mathrm{CS}_{1}$ as described by Eq. (5), the compatibility equations. Forces and moments developed in the spring at $\mathrm{CS}_{1}$ transfer to $\mathrm{CS}_{0}$ through the link as described by Eq. (6), the equilibrium equations. Both the compatibility equations and the equilibrium equations are readily expressed in terms of six-dimensional vectors. In so doing, a single matrix, the transformation matrix, satisfies both the compatibility equations in Eq. (7) and the equilibrium equations in Eq. (8). The Appendix shows the derivation and other details important for implementing the transformation matrix in a computer program.

$$
\begin{gathered}
d \boldsymbol{\delta}_{1}=\mathbf{R}_{1 / 0} \cdot\left(d \boldsymbol{\delta}_{0}+d \boldsymbol{\theta}_{0} \times \mathbf{r}_{0}\right) \\
d \boldsymbol{\theta}_{1}=\mathbf{R}_{1 / 0} \cdot d \boldsymbol{\theta}_{0} \\
\mathbf{f}_{0}=\mathbf{R}_{0 / 1} \cdot \mathbf{f}_{1} \\
\mathbf{m}_{0}=\mathbf{R}_{0 / 1} \cdot \mathbf{m}_{1}+\mathbf{r}_{0} \times\left(\mathbf{R}_{0 / 1} \cdot \mathbf{f}_{1}\right) \\
{\left[\begin{array}{c}
d \boldsymbol{\delta}_{1} \\
-- \\
d \boldsymbol{\theta}_{1}
\end{array}\right]=\mathbf{T}_{0 / 1}^{T} \cdot\left[\begin{array}{c}
d \boldsymbol{\delta}_{0} \\
--- \\
d \boldsymbol{\theta}_{0}
\end{array}\right]} \\
{\left[\begin{array}{c}
\mathbf{f}_{0} \\
--- \\
\mathbf{m}_{0}
\end{array}\right]=\mathbf{T}_{0 / 1} \cdot\left[\begin{array}{c}
\mathbf{f}_{1} \\
-- \\
\mathbf{m}_{1}
\end{array}\right]}
\end{gathered}
$$

\subsection{Force-deflection relations for systems of springs}

The concept of parallel and series combinations of components is useful in a number of engineering disciplines. For example, electrical resistor analogies are common in fluid mechanics and heat transfer. In mechanics, the springs can be multi-dimensional and aligned in different directions, provided that the loads and deflections for all the springs are represented in the same CS. Then the springs in parallel experience the same deflection and their loads add, while springs in series experience the same load and their deflections add. For linear or linearized springs, stiffness matrices are added to represent parallel combinations and compliance matrices are added to represent series combinations. Mixed combinations of parallel and series springs require like groups to be combined first then inverted as necessary to combine with other groups.

The $[6 \times 6]$ transformation matrix handles the task of converting force-moment and differential translation-rotation vectors to the proper CS. The block diagram in Fig. 4 shows the required process flow for parallel springs (a) and series springs (b) [16]. The subscript $i$ indicates the spring number, and there are as many loops in the diagram as there are springs. Eq. (9) shows the equivalent stiffness matrix $\mathbf{K}_{0}$ for springs in parallel; the inverse is the equivalent compliance matrix. Eq. (10) shows the equivalent compliance matrix $\mathbf{C}_{0}$ for springs in series; the inverse is the equivalent stiffness matrix. This technique applies equally well to inertial and damping quantities expressed with six-dimen- 


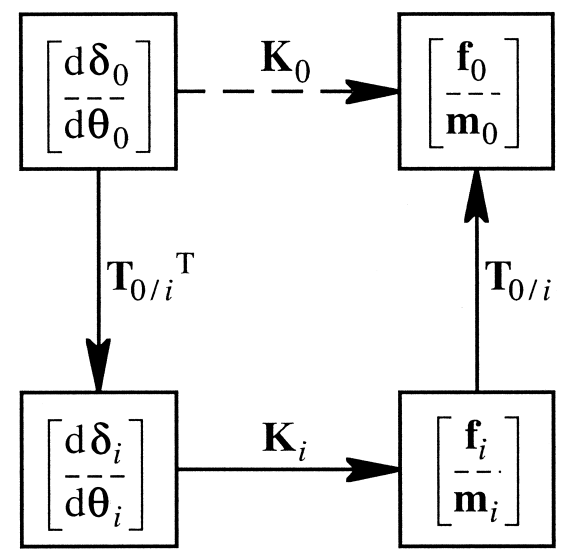

(a)

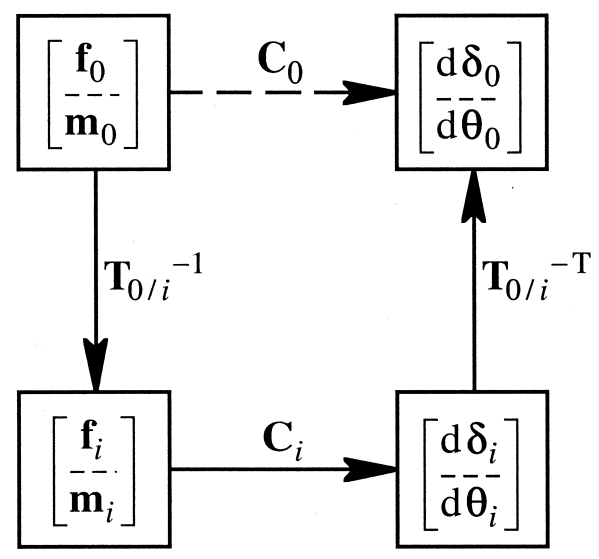

(b)

Fig. 4. The diagram shows the required process flow for parallel springs (a) and series springs (b), after Strang [16].

sional vectors. When applied to an inertia matrix, for example, it is a generalized form of the parallel axis theorem.

$$
\begin{aligned}
& \mathbf{K}_{0}=\sum_{i} \mathbf{T}_{0 / i} \cdot \mathbf{K}_{i} \cdot \mathbf{T}_{0 / i}{ }^{T} \\
& \mathbf{C}_{0}=\sum_{i} \mathbf{T}_{0 / i}{ }^{-T} \cdot \mathbf{C}_{i} \cdot \mathbf{T}_{0 / i}{ }^{-1}
\end{aligned}
$$

It is useful to review the steps in the system modeling problem before moving on to optimization. The analyst must identify the number and combination of springs that make up the system, typically six parallel springs for a kinematic coupling. Then the analyst defines the stiffness or compliance matrix (or function) for each spring in its own CS. Although somewhat arbitrary, the analyst must choose a convenient base CS with which to represent the system as an equivalent spring. With all the CS's chosen, the analyst can determine the instruction set describing the position and orientation of each spring CS to the base CS. Usually some of the instructions become parameters in the optimization study. Finally the analyst must assemble the equation for the equivalent spring in the manner of Eqs. (9) and (10). Since the model is completely parametric, both the configuration, represented by the local CS's, and the spring elements may be optimized as the analyst sees fit.

\section{Optimization criteria}

The optimization criteria provide meaningful, quantitative measures with which to evaluate and optimize the kinematic coupling design. The optimization process involves varying some subset of the coupling parameters in a way that makes one or more of the optimization criteria maximum or minimum as the case may be. From the user perspective, the main decision is which one(s) to use, and this will depend on the main requirements for the particular application. The four criteria presented here should suffice for most application and others could be created for special purposes. In setting up the algorithms to compute the optimization criteria, there is a decision regarding how to combine typically six values computed for each constraint. Since this aspect applies to all, it will be presented now in advance of the individual criteria.

The generalized mean, defined in Eq. (11), gives the user complete flexibility in emphasizing larger elements in the combination over the smaller elements or vice versa by adjusting the value of the exponent $p$. For example, using $p=2$ is the familiar root mean square (RMS), which places somewhat greater emphasis on larger elements. In the limit as $p \rightarrow \infty$, the generalized mean picks only the maximum element and ignores the rest. A negative exponent changes the elements to reciprocals, thus placing greater emphasis on smaller elements. In between positive and negative as $p$ $\rightarrow 0$, the generalized mean gives the same result as the geometric mean. This indicates that the geometric mean is neutral, placing no emphasis on large or small elements. In practice, however, we typically use the maximum or minimum element depending on the optimization criterion.

$$
\operatorname{gen} \_\operatorname{mean}(\mathbf{v}, p)=\left\{\frac{1}{n} \sum_{i=1}^{n}\left|v_{i}\right|^{p}\right\}^{1 / p}
$$

\subsection{Maxwell's criterion}

Although not closely tied to a physical measure, Maxwell's criterion is simple to implement and it demonstrates techniques used in more specialized optimization criteria. It states that each constraint should be aligned to the local direction of motion allowed by the five other constraints, assuming that they remain engaged and are free to slide. To test this criterion, each constraint is released individually to discover the direction of motion at the released constraint compared to the actual constraint direction. This test is demonstrated in Fig. 5 on a symmetric three-vee coupling. The inner product (or dot product) between the direction of motion and the constraint direction provides a convenient scalar indicator of the alignment. The vector directions are given unit length so that perfect alignment produces a maximum inner product of one. There will be one inner product for each constraint, and the objective is to maximize all six. For this symmetric coupling, the inner product is the same 

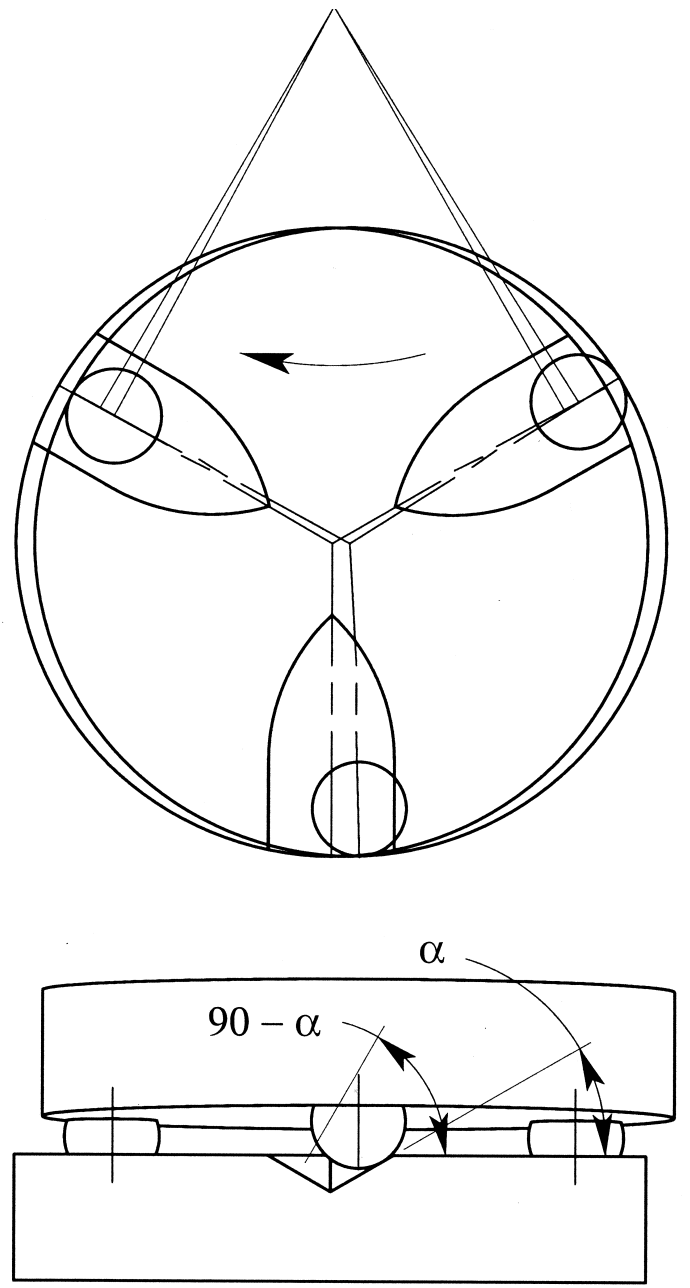

Fig. 5. The three-vee coupling slides on five constraints producing rotation about an instant center shown in the top view and also about an axis through the two seated balls.

for each of the six constraints and equal to $\cos \left(90^{\circ}-2 \alpha\right)$, which is maximum at $\alpha=45^{\circ}$.

Computing the inner product for each constraint turns out to be a rather simple process. The constraint direction is always along the $z$-axis of the local CS by convention. Then the inner product reduces to the $z$-component of a unit vector along the direction of motion at the released constraint, also in the local CS. The equivalent stiffness matrix computed with only five constraints will have a singular direction with zero stiffness. This direction in the base $\mathrm{CS}_{0}$ is given by the eigenvector of the stiffness matrix corresponding to the zero eigenvalue. Multiplying $\mathbf{T}_{0 / \mathrm{i}}{ }^{\mathrm{T}}$ by the six-dimensional eigenvector transforms the eigenvector to the local $\mathrm{CS}_{\mathrm{i}}$. The translation part of this transformed singular direction is the direction of motion at the constraint, but it may not be unit length. Dividing the $z$-component by the length gives the inner product for that particular constraint. This procedure is repeated for each of the five remaining constraints, and the minimum value is typically used in the design optimization. Maxwell's criterion, being computed from the singular directions, is a function only of the configuration of constraints, not their stiffness. Some of the other optimization criteria will involve the constraint stiffness.

\subsection{Maximizing the modal frequency}

The modal frequencies of a dynamic system are computed from the mass and stiffness matrices using the generalized eigenvalue algorithm. This requires the continuous system to be represented by a discrete number of degrees of freedom, for example, as done in the finite element method with interpolating functions. As a first approximation, the coupling and payload are assumed to be rigid so that the mass matrix will be six dimensional like the stiffness matrix. The mass is recorded in the first three diagonal elements, the $[3 \times 3]$ inertia matrix is recorded in the lower diagonal block, and the rest are zeros. The $[3 \times 3]$ inertia matrix and the newly created $[6 \times 6]$ mass matrix both apply about the mass centroid. Either the mass matrix or the stiffness matrix must be reflected to the CS of the other before applying the generalized eigenvalue algorithm. The six eigenvalues that it computes are equal to the squares of the modal frequencies in radians per second if the mass and stiffness are in consistent units. The minimum value is typically used in the design optimization, but the coupling optimized just for frequency may not function well in other respects such as sliding to a centered position. Once a balanced optimum is found, finite element analysis can be used to improve the accuracy of the modal frequencies.

Continuing the example of the symmetric three-vee coupling, a mass element will be placed at the center of stiffness and given principal moments of inertia equal to $m R_{m}{ }^{2}$, where $m$ is the mass and $R_{m}$ is the radius of gyration. Eq. (12) gives the stiffness matrix, where $k$ is the stiffness of each constraint and $R_{k}$ is the radius to each constraint. The angle $\alpha$ is the parameter to optimize and is defined the same as before (see Fig. 5). The modal frequencies, in this case, are determined simply by dividing the diagonal elements of $\mathbf{K}$ by those of $\mathbf{M}$ with the results shown in Eq. (13).

$$
\begin{aligned}
& \mathbf{K}=\operatorname{diag}\left(\left[\begin{array}{c}
3 k \sin ^{2}(\alpha) \\
3 k \sin ^{2}(\alpha) \\
6 k \cos ^{2}(\alpha) \\
3 k R_{k}^{2} \cos ^{2}(\alpha) \\
3 k R_{k}^{2} \cos ^{2}(\alpha) \\
6 k R_{k}^{2} \sin ^{2}(\alpha)
\end{array}\right]\right) \\
& \boldsymbol{\omega}=\left(\frac{3 k}{m}\right)^{\frac{1}{2}}\left[\begin{array}{c}
\sin (\alpha) \\
\sin (\alpha) \\
2 \cos (\alpha) \\
\gamma \cos (\alpha) \\
\gamma \cos (\alpha) \\
2 \gamma \sin (\alpha)
\end{array}\right] \quad \gamma=\frac{R_{k}}{R_{m}}
\end{aligned}
$$

Only a few degrees of freedom matter in the optimization depending on the radius ratio $\gamma$. For example when $\gamma$ is 
small, the angular degrees of freedom will limit the modal frequencies. This is one of three regimes to evaluate as indicated in Eq. (14). The center regime finds the balance between $x-y$ linear and $x-y$ angular degrees of freedom. The last regime is governed by the linear degrees of freedom. It is useful to check the consequence of using a suboptimal angle, say $\alpha=45^{\circ}$, when one of the outer regimes is optimal. In either case, the minimum modal frequency would be reduced a factor 0.866 from the optimal condition. This is not very significant although other applications could be somewhat worse, for example, if the center of mass lies distant from the center of stiffness.

$$
\alpha=\left\{\begin{array}{lc}
\arctan \left(2^{-1 / 2}\right) \cong 35^{\circ} & \gamma<2^{-1 / 2} \\
\arctan (\gamma) & 2^{-1 / 2} \leq \gamma \leq 2^{1 / 2} \\
\arctan \left(2^{1 / 2}\right) \cong 55^{\circ} & \gamma>2^{1 / 2}
\end{array}\right.
$$

\subsection{Minimizing frictional nonrepeatability}

Repeatable engagement is frequently a driving requirement for and motivation to use a kinematic coupling. Friction between the contacting surfaces acting on the compliance of the coupling is a main contributor to nonrepeatable behavior [5]. Eq. 15 provides a simple order-of-magnitude estimate for nonrepeatability that came from earlier work $[13,14]$. While the estimate provides the general trends for the given parameters, it lacks any information about the configuration. ${ }^{6}$ For example, the frictional force for any particular direction of sliding is proportional to the applied load $P$, the coefficient of friction $\mu$ and the configuration of constraints. The effective stiffness of a constraint, in the manner of Maxwell, is affected by the angle of the constraint compared to the direction of sliding it constrains. Thus both contributors to nonrepeatability (friction and compliance) are affected by the configuration of constraints. Furthermore, the magnitude of nonrepeatability is likely to vary among the six sliding directions and directions in between.

$$
\rho \equiv \frac{f}{k} \approx \mu\left(\frac{2}{3 R}\right)^{1 / 3}\left(\frac{P}{E}\right)^{2 / 3}
$$

To make this problem tractable, an assumption must be made regarding the directions to check for nonrepeatability. We will use the six singular directions found for Maxwell's criteria assuming that these well represent the nonrepeatability for all other directions. This sample may not bound the worse-case nonrepeatability, but the main purpose is to represent the character of the coupling so it may be optimized. The next section will provide logic that lends credence to this assumption.

This method begins as with Maxwell by finding the singular direction of a five-constraint stiffness matrix. Again the actual stiffness of each constraint is not important for this step but it will become important subsequently. The singular direction is used in computing the frictional force-

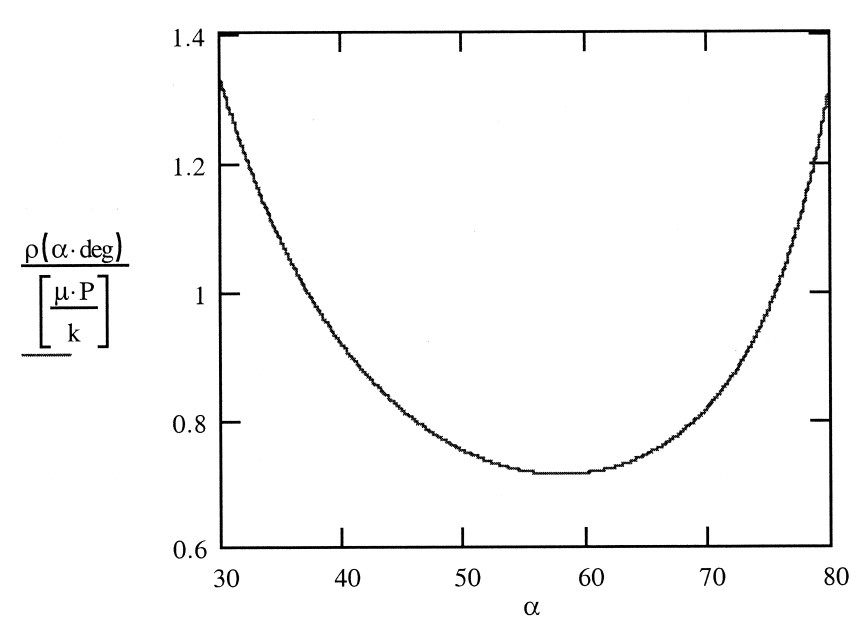

Fig. 6. The effect of the vee angle $\alpha$ on the repeatability of the symmetric three-vee coupling has a minimum of 0.71 at $58^{\circ}$.

moment vector, which acts on the full compliance matrix resulting in a translation-rotation vector of nonrepeatability. This vector is given in the same CS as the compliance matrix, which may not be the functional point of the coupling, for example, a tool point. It is straightforward to transform the nonrepeatability vector to another CS and compute the vector magnitude, usually only the translation part. This procedure is repeated for each of the five remaining constraints, and the maximum value is typically used in the design optimization.

Computing the frictional force-moment vector is a new aspect in this method worth more detailed explanation. The singular direction must first be transformed to each local CS to obtain the direction of translation at each constraint. The local friction force will lie in the $x-y$ plane and be equal in magnitude to the coefficient of friction times the normal force at the constraint. ${ }^{7}$ A simplifying step works under the assumption that the normal forces remain at the values calculated for equilibrium without friction. In effect, friction causes a nonrepeatability in the load vector required to bring the coupling to the ideal engagement point. This is the frictional force-moment vector, which is computed by transforming the six local friction forces to the base CS of the coupling and then adding. Multiplying the compliance matrix by this vector gives the nonrepeatability vector.

For the symmetric three-vee coupling in Fig. 5, the dominant component of nonrepeatability at the center will be horizontal. Neglecting the other components and assuming the coupling is symmetrically loaded, Eq. (16) gives the nonrepeatability as a function of configuration, represented by $\alpha$, and other terms $\mu, P$ and $k$. The effect of the configuration is plotted in Fig. 6, which shows a minimum of 0.71 at $\alpha=58^{\circ}$. The estimate in Eq. (15), corresponding to a factor 1 in the figure, is somewhat conservative for the symmetric three-vee coupling having a nearly optimal vee angle. 


$$
\rho \cong \frac{\mu P}{18 k \sin ^{2} \alpha \cos \alpha}(2 \sqrt{3}+\cos \alpha+\sin 2 \alpha)
$$

\subsection{Maximizing the limiting coefficient of friction}

The extreme case of a nonrepeatable coupling is one that fails to slide to the centered position. It becomes centered when all constraints are fully engaged although it may not go to the ideal center where the potential energy is minimal. The coupling will center if the real coefficient of friction is less than the limiting value when the net centering force goes to zero. The coupling optimized for centering will have the maximum margin obtained by maximizing the limiting coefficient of friction over the real coefficient of friction. In practice, a margin of 3:1 is reasonable to obtain and quite sufficient.

When a coupling is brought together initially off center, the constraints engage sequentially as the coupling seeks a path to center. This path becomes better defined as more constraints engage. For example, five constraints allow the coupling to slide along one well-defined path. Four constraints allow motion over a two-dimensional surface of paths and so forth. As each new constraint engages, it directs the coupling along a more resistive path to center. In other words, the coupling looses the freedom to follow the least resistive path. This means that a path with five constraints engaged will set the limiting coefficient of friction, and there are only six such paths to analyze. These are the six singular directions found for Maxwell's criterion and the ones used to sample the nonrepeatability of the coupling. The method used to compute the frictional forces is essentially the same as before except that the coefficient of friction is now a variable to be solved from the equation for the constraint not engaged, since its reaction force must be zero for the coupling to be on the verge of sliding. ${ }^{8}$ This procedure is repeated for each of the five remaining constraints, and the minimum value is typically used in the design optimization.

When the symmetric three-vee coupling in Fig. 5 is loaded with a nesting force, a centering force develops tending to slide the coupling along the singular direction. The off-center vee transforms its share of the nesting force (assumed to be one-third in this example) into a centering moment about the instant center. Eq. (17) describes the centering force at the center of the coupling due to this moment as a function of the angle $\alpha$ and the coefficient of friction $\mu$. The limiting coefficient of friction corresponds to the threshold of sliding when the net centering force or moment is zero. In this example, the six sliding directions are symmetrically identical and equally loaded so there is only one equation to solve for $\mu$ as a function of $\alpha$. Reviewing the numerical results in Table 1 , a $60^{\circ}$ angle for the three-vee coupling is very close to optimal. This result is consistent with that found for minimum frictional nonre-
Table 1

The limiting coefficient of friction for symmetric three-vee and threetooth couplings varies with the inclination angle of the contact surfaces. The margin for centering is of order 3:1 using a typical coefficient of friction of 0.10 to 0.12 .

\begin{tabular}{lccccc}
\hline Angle of Inclination $\alpha$ & $45^{\circ}$ & $50^{\circ}$ & $55^{\circ}$ & $60^{\circ}$ & $65^{\circ}$ \\
Three-Vee Coupling & 0.317 & 0.338 & 0.354 & 0.364 & 0.365 \\
Three-Tooth Coupling & 0.319 & 0.339 & 0.351 & 0.352 & 0.339 \\
\hline
\end{tabular}

peatability, but it has slightly higher contact stress than the result found for Maxwell's criterion, $\alpha=45^{\circ}$.

$$
\frac{f_{c}}{f_{n}}=\frac{\sin \alpha-\mu \cos \alpha}{2(\cos \alpha+\mu \sin \alpha)}-\frac{\sqrt{3 \mu}}{3 \cos \alpha}
$$

Table 1 also gives the results for a new kinematic coupling called the three-tooth coupling [17]. Shown in Fig. 7, the three-tooth coupling forms three theoretical lines of contact between cylindrical teeth on one member and flat teeth on the other member. ${ }^{9}$ Each line constrains one translation and one rotation to give a total of six constraints; however, it is somewhat simpler to model this as six translational constraints each centered on a tooth face.

\section{Kinematic mounts for NIF optics assemblies}

The National Ignition Facility (NIF) when completed in 2003 will be the world's most powerful laser system and the first facility capable of achieving nuclear fusion and energy gain in a laboratory. Being able to create

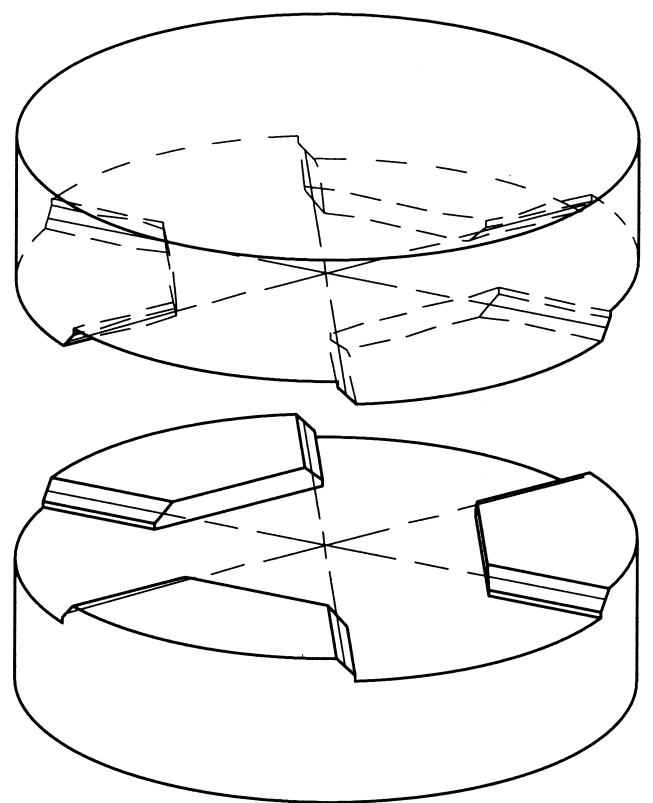

Fig. 7. The three-tooth coupling forms three theoretical lines of contact between cylindrical teeth on one member and flat teeth on the other member. 


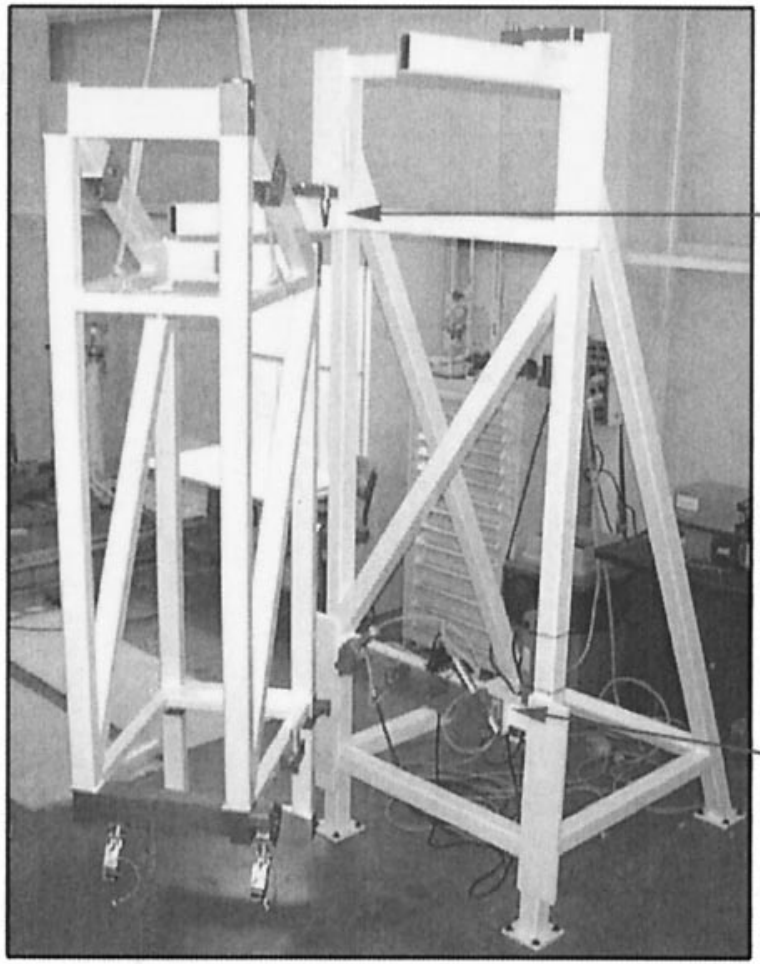

(b)

(a)

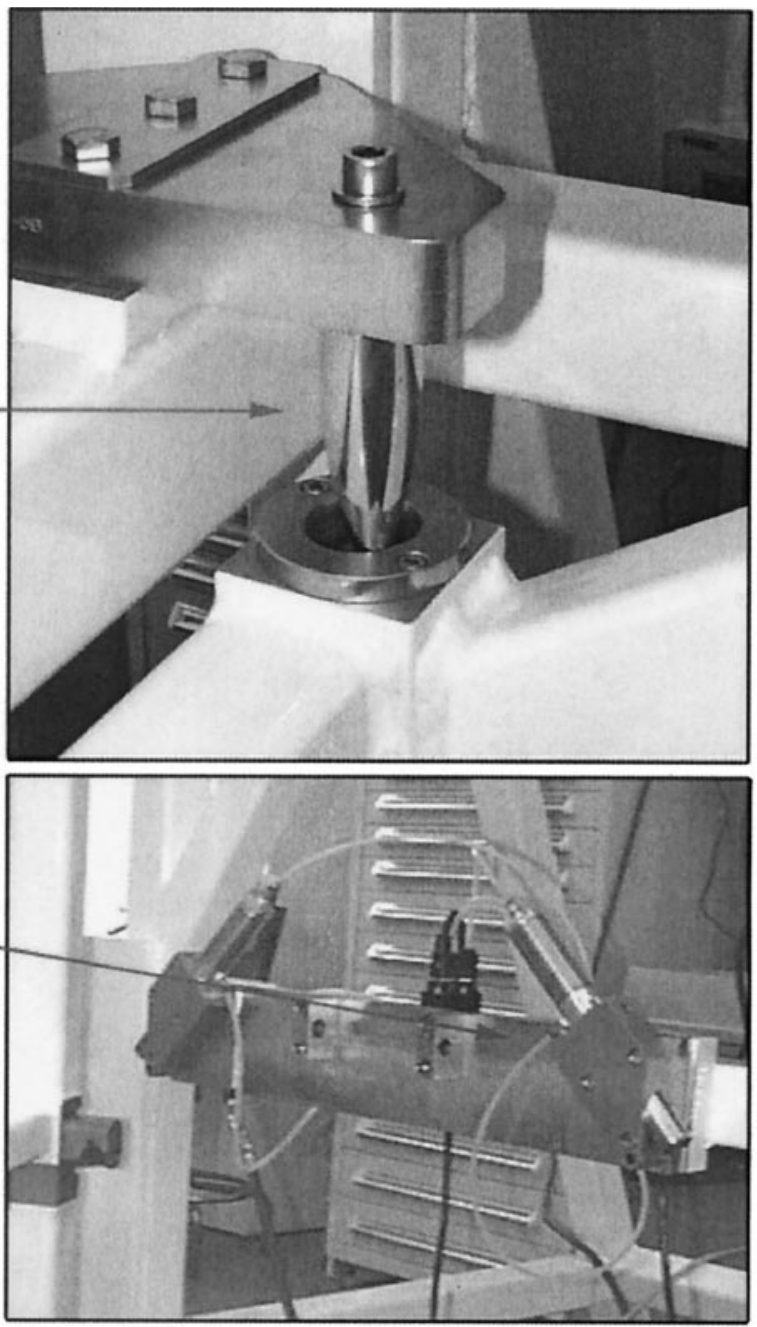

Fig. 8. The prototype LRU in (a) is over $2.5 \mathrm{~m}$ tall, and when mounted in the NIF periscope structure, the lowest point will be $3.6 \mathrm{~m}$ to $5.5 \mathrm{~m}$ above the floor, depending in its location in a staircase-like structure. The upper mount has two pin-slot constraints like the one shown in (b) partially engaged. The $\varnothing 35 \mathrm{~mm}$ pins attach to the structure. In (c), two vee blocks on the LRU engage two $\varnothing 32 \mathrm{~mm}$ actuated pins on the structure to form the lower mount.

conditions more extreme than the center of the Sun, it will have far-reaching implications for fusion energy and a variety of scientific fields. The architecture for the NIF laser system is heavily influenced by the need to routinely replace damaged optics in a very clean and inert atmosphere. A concept was adopted where any optics assembly, known as a line replaceable unit or LRU, could be installed or removed by a semi-automated transportation canister from underneath the laser. The canister docks to the laser structure, establishes a pressure-tight seal, removes an access panel, and installs the LRU with a straight-line lift. This concept for the NIF requires many hundreds of large kinematic couplings to handle LRUs with the transportation canister and to mount them within the laser structures. The kinematic mount between the LRU and laser structure, although based on three vees, deviated from our experience sufficiently to motivate an optimization using the limiting coefficient of friction. Fig. 8 shows this design on a prototype LRU, which is temporarily separated from the support structure to show the kinematic mount.

The basic configuration of the kinematic mount is a three-vee coupling with one widely spaced vee at the top and two vees near the bottom. Fig. 9 shows the locations and orientation of the six constraints in four different views. Configuring the mounting points to lie in a vertical plane gives the most favorable aspect ratio and accommodates the dense packing of LRUs. Furthermore, finite element analysis showed that the torsional mode of the frame would be a limitation to vibrational stability, assuming zero friction in the constraints. Placing the instant center of the upper vee near the principal axis of the LRU reduces the inertia in the torsional mode and increases the frequency. A more significant motivation for the wide vee is the frictional constraint against rotation, which is an order of magnitude stiffer than the LRU frame. The potential static twist in the frame due to friction is much less than the requirement for initial alignment. 

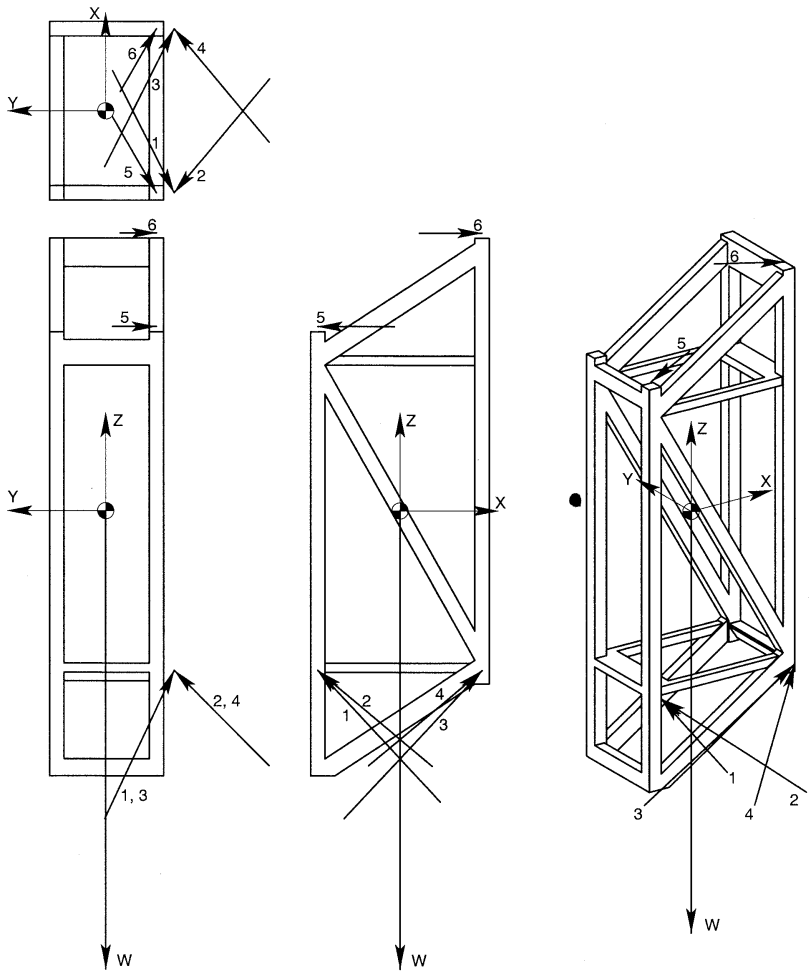

Fig. 9. Orthographic and isometric views of one LRU. The arrows numbered 1-6 represent the six constraints that support the assembly. The arrows are proportional to the reaction forces.

Physically, the upper vee consists of two pin-slot constraints that passively engage as the LRU lifts into place. As Fig. 10 shows, each constraint consists of a tapered pin attached to the structure and a slotted receiver at the top of the LRU. Conversely, the lower mount is active and formed by two vee blocks on the LRU that can travel past retracted pins on the structure. As Fig. 11 shows, the pins extend to receive the vee blocks and support the weight of the LRU, which ranges from 300 to $600 \mathrm{kgf}$ depending on LRU type. Each pin mechanism is actuated by a pneumatic cylinder and for safety reasons is incapable of retracting under the weight of the lightest LRU. Due to the angle of the pin, the load is primarily compressive across its $32 \mathrm{~mm}$ diameter.

\subsection{A graphical approach to multi-variable optimization}

A parameterized model of the NIF LRU was developed using the methods described in this paper. The positions of the constraints were set largely by geometric considerations, but the angles of the constraints were controlled by four variable parameters to be optimized: 1 ) the pin angle of the lower mount, 2) the outside vee angle of the lower mount, 3) the inside vee angle of the lower mount, and 4) the slot angle of the upper mount. At the time, only the limiting coefficient of friction criterion had been developed to optimize centering ability. Rather than using an optimization algorithm, the criterion was plotted
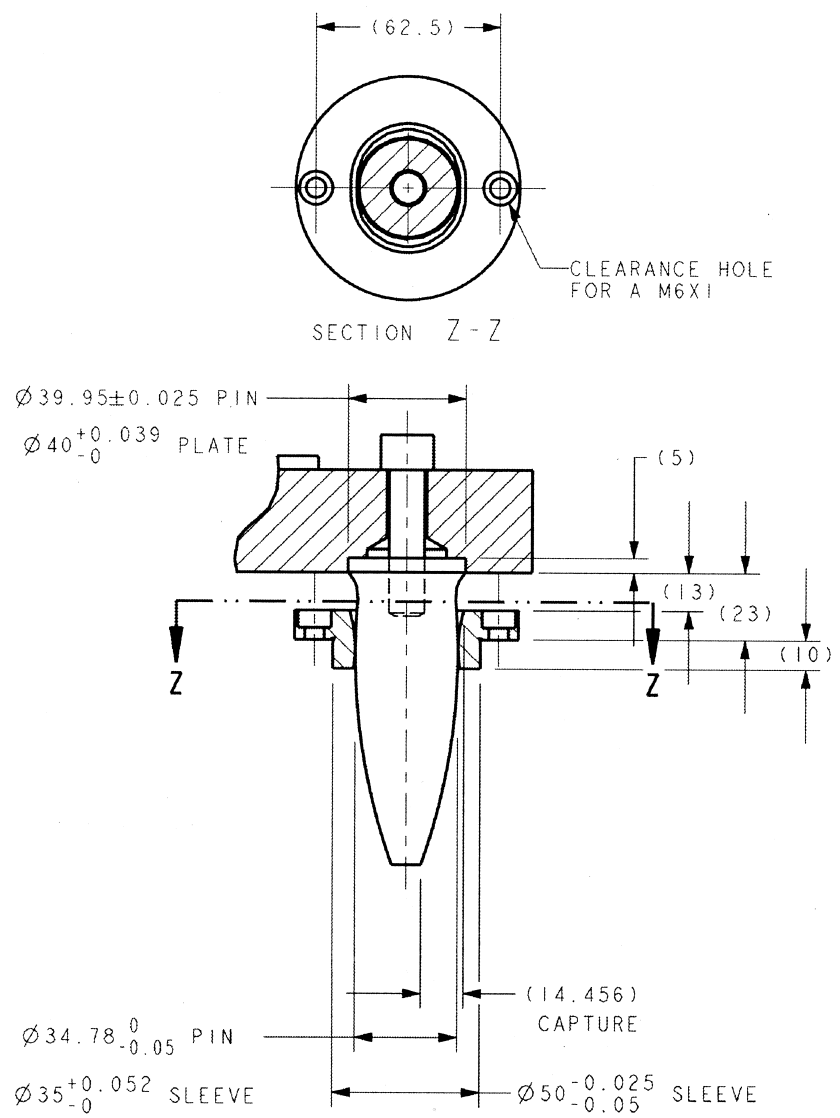

Fig. 10. The slotted receiver at the top of the LRU engages the tapered pin on the structure with approximately $15 \mathrm{~mm}$ of radial capture range.

in such a way that clearly shows the sensitivity to each parameter and how to converge to the optimum within a few iterations. This graphical approach leads to a better

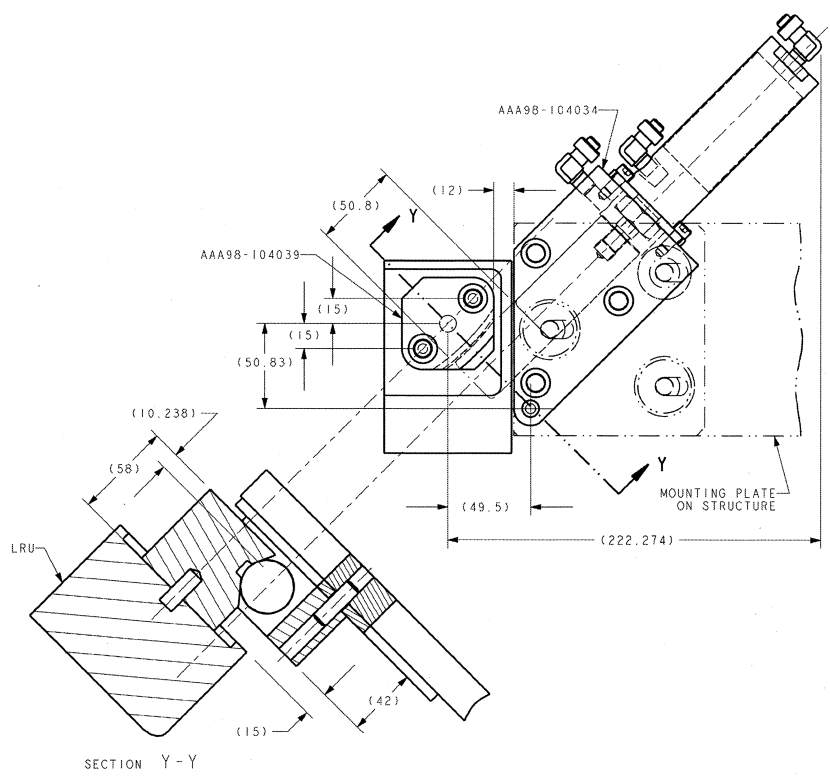

Fig. 11. The pneumatically actuated pin extends underneath the vee block to support the LRU. 

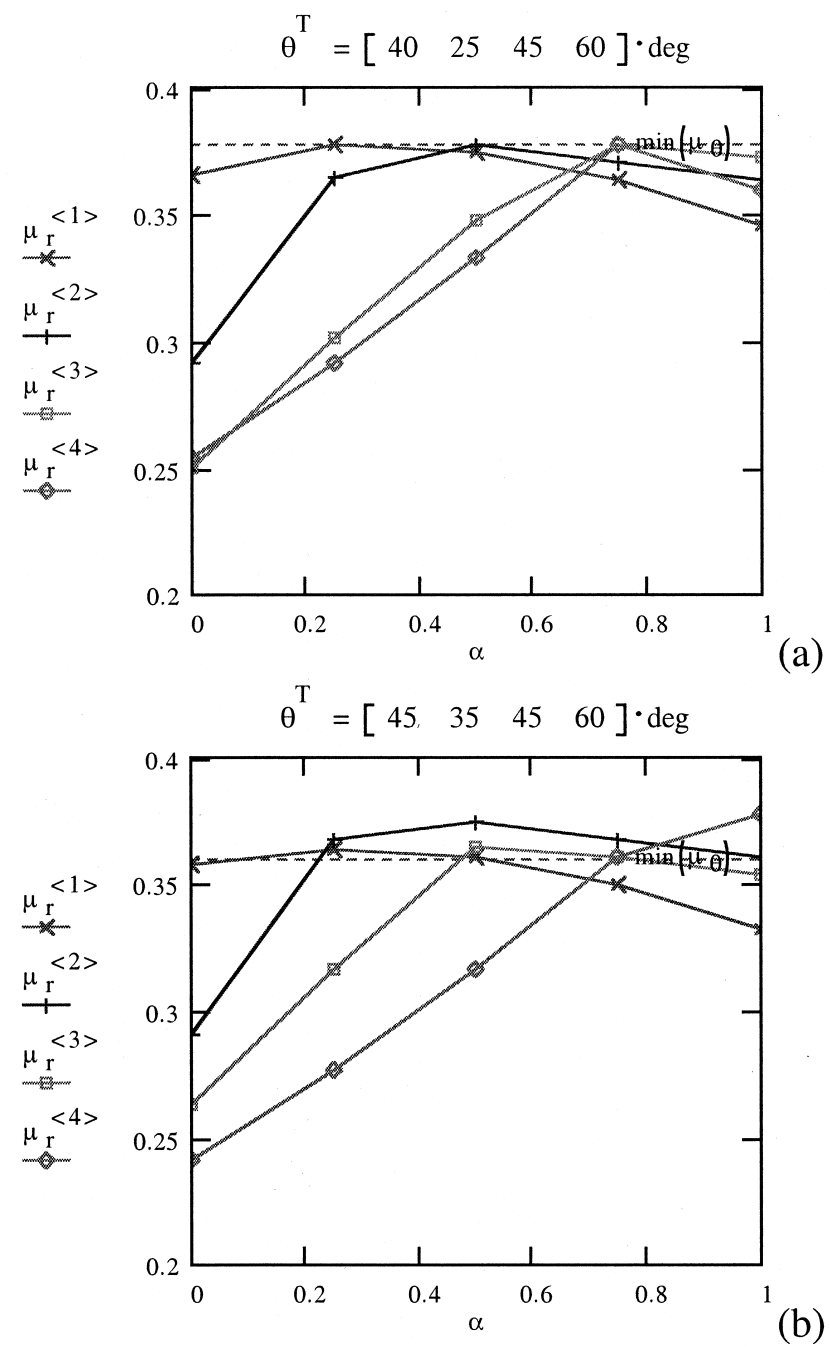

Fig. 12. Limiting coefficient of friction versus a normalized range of model parameters. Each curve corresponds to one individually varying parameter while the others are held at nominal values. The horizontal dashed line indicates the value for the nominal parameter set. The graph in (a) shows the optimal configuration while (b) is suboptimal. In this example, the markers occur in $5^{\circ}$ increments of the angle parameters.

understanding of the system and the tradeoffs between parameters.

To begin the graphical optimization, the analyst chooses a nominal value for each parameter and the range that it will vary. Then for each variable parameter, the optimization criterion is computed over the range while holding all other parameters at their nominal values. This leads to a set of curves showing how the optimization criterion varies with each parameter about the nominal design. If the ranges are quite different, the curves may be plotted versus a normalized parameter range as is done in Fig. 12 to give a compact horizontal axis. Fig. 12 shows two graphs for the NIF LRU with slightly different nominal parameters as indicated above each graph. The horizontal dashed line indicates the limiting coefficient of friction for the nominal parameter set. The optimal parameter set is apparent in (a) because a change to any one parameter reduces the limiting coefficient of friction. It is useful to observe the suboptimal parameter set in (b). Curves that extend above the dashed line generally indicate that improvement is possible. The analyst would adjust the nominal value toward the peak for one or more curves, attempting to maximize the criterion in this case. However, since changing just one parameter can change the shape of several curves, it is usually best to make partial steps toward the peaks, as an optimization algorithm would.

\section{Summary}

Designers of kinematic coupling now have a set of quantitative measures with which to evaluate, compare or optimize arbitrarily complex configurations of six constraints. The first measure comes directly from the recommendation of Maxwell, to align constraints to the directions that they constrain. Think of this as efficiency. The second measure is rather common today given the ease with which modal frequencies are computed with finite element programs. Modal frequency may be an important factor but probably not the best choice to drive an optimization. The third and fourth measures have clear physical meaning and usually are driving requirements for kinematic couplings. Minimize the frictional nonrepeatability when positioning repeatability is critical. Maximize the limiting coefficient of friction when reliable centering is critical. These measures are conveniently implemented in parametric models using six-dimensional springs and $[6 \times 6]$ transformation matrices. This modeling approach is explained for completeness and because it is useful in other applications such as flexure systems. Space does not permit including a Mathcad ${ }^{\mathrm{TM}}$ program developed for optimal kinematic coupling design but interested readers may obtain hard or soft copies by contacting the authors.

\section{Notes}

1. The instantaneous center of rotation (or instant center for short) is a useful visual aid in understanding the motion that combinations of constraints allow. For example in Fig. 2, Vee 1 and 2 constrain four degrees of freedom and allow rotation about two axes. One axis passes through the center of each ball and the other passes through the instant center perpendicular to the plane of the vees. When engaged, Vee 3 constrains rotation about these two axes. See page 115 .

2. In addition, one reviewer of this paper described an essentially identical graphical method by Wittgen, but the authors were unable to locate this reference. See page 115 .

3. One reviewer of this paper pointed out that the effective hinge axis of the flexure should coincide 
with the effective center of curvature for the pair of contacting surfaces. Otherwise there will be a second-order error in repeatability of the coupling depending on the degree of bending in the flexure. See page 115 .

4. Two CSs that do not have a common origin may or may not require accounting for the offset depending on the quantity being rotated. For example position vectors require accounting for the offset while angular velocity vectors do not. See page 116.

5. Paul [15] derives a method using HTMs to transform differential translations and rotations from one CS to another. He also shows that the resulting expressions may be put in the form of a six-dimensional matrix equation. Later he extends this result to forces and moments using the principle of virtual work. He does not, unfortunately, give the matrix a name. Although technically a homogeneous transformation matrix, we use [6 $\mathrm{x} 6]$ transformation matrix to avoid confusion with the [4 x 4] HTM. See page 117.

6. The estimate is derived as if the couplings compliance in all directions is equal to a single Hertzian contact carrying a load $P$ and having a relative radius $R$ and elastic modulus $E$. See page 120 .

7. The constraint released to obtain the singular direction should have a frictional force reduced by the ratio of $x-y$ motion divided by $x-y-z$ motion. This ratio will be one for the other five constraints. See page 120 .

8. Since the eigenvalue algorithm may compute a siding direction that is into or out of engagement, the computed coefficient of friction may be correspondingly positive or negative. Using the absolute value solves this problem. Nature selects the direction into engagement yielding a positive coefficient of friction, assuming the coupling is designed to be stable. This condition is checked by seeing that all six constraints are in compression at equilibrium. See page 121 .

9. The form of the teeth along the three lines of contact must be straight to a small fraction of the calculated Hertz deflection to achieve continuous contact and fairly uniform stress. Other form tolerances may be relatively loose and are driven primarily by the accuracy required of the coupling rather than its repeatability. See page 121.

\section{Acknowledgments}

The authors acknowledge the valued comments from the reviewers. Their specific comments have been added as footnotes. Their general comments led to better clarity and completeness.

This work was performed under the auspices of the U.S. Department of Energy by the University of California Law-

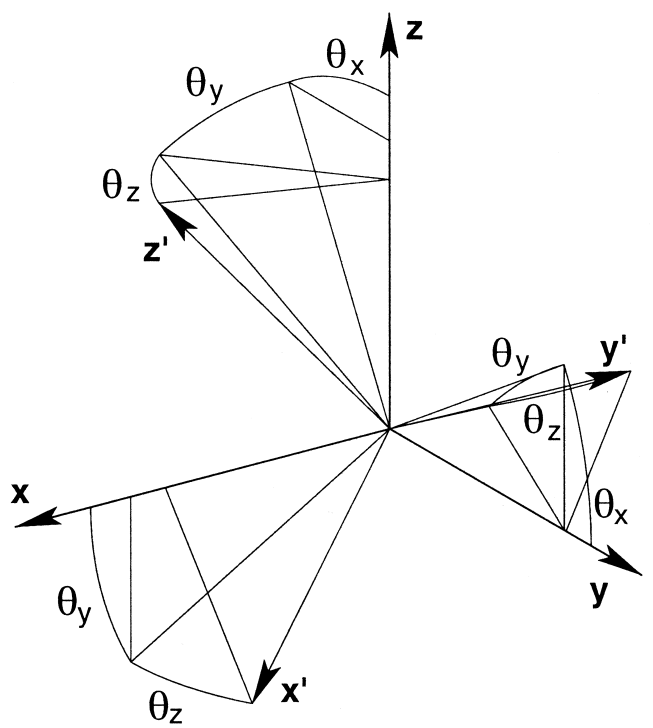

Fig. 13. Any arbitrary orientation can be represented as a sequence of three single-axis rotations such as this one about base $x, y$, and $z$ axes.

rence Livermore National Laboratory under contract No. W-7,405-Eng-48

\section{Appendix}

The $[6 \times 6]$ transformation matrix is constructed from blocks of more elementary $[3 \times 3]$ matrices: the rotation matrix, the cross product matrix, the identity matrix and zeros. Understanding the rotation matrix is key to understanding the $[6 \times 6]$ transformation matrix so it is addressed first. Recall that the columns of the rotation matrix express the $x, y, z$ axes, respectively, of the rotated CS with respect to the base CS. The rotation matrix is easiest to derive when the rotation occurs about any one coordinate axis. Eqs. (18), (19), and (20) give, respectively, the rotation matrices for separate rotations about $x$, $y$ and $z$ axes. Being an orthogonal CS, the rotation matrix is orthonormal by definition and the transpose operation provides the inverse. In addition, these single-axis rotation matrices are skew symmetric, so the inverse transformation is simply the reverse rotation. Sequential rotations are easy to compute by multiplying single-axis rotation matrices in the proper order.

$$
\begin{aligned}
& \mathbf{R}_{x}\left(\theta_{x}\right)=\left[\begin{array}{ccc}
1 & 0 & 0 \\
0 & \cos \theta_{x} & -\sin \theta_{x} \\
0 & \sin \theta_{x} & \operatorname{cox} \theta_{x}
\end{array}\right] \\
& \mathbf{R}_{y}\left(\theta_{y}\right)=\left[\begin{array}{ccc}
\cos \theta_{y} & 0 & \sin \theta_{y} \\
0 & 1 & 0 \\
-\sin \theta_{y} & 0 & \cos \theta_{y}
\end{array}\right] \\
& \mathbf{r}_{z}\left(\theta_{z}\right)=\left[\begin{array}{ccc}
\cos \theta_{z} & -\sin \theta_{z} & 0 \\
\sin \theta_{z} & \cos \theta_{z} & 0 \\
0 & 0 & 1
\end{array}\right]
\end{aligned}
$$




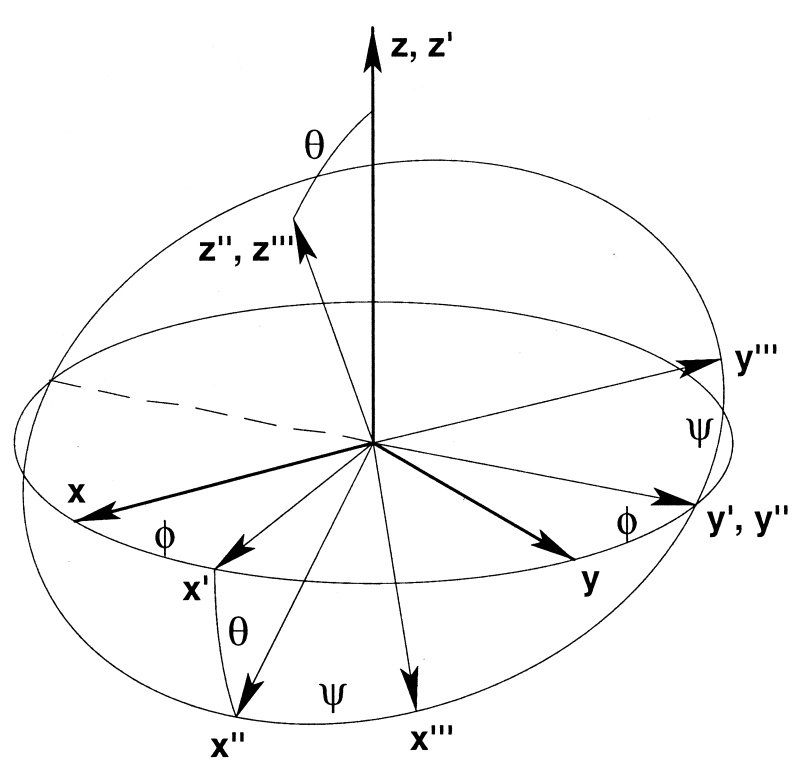

Fig. 14. Euler angles describe an arbitrary orientation with sequential rotations $\phi, \theta, \psi$ about body $z, y^{\prime}, z^{\prime \prime}$ axes.

A natural sequence of rotations and the one depicted in Fig. 13 is rotation about the base axes in the order $x, y, z$. Remembering to pre multiply for base coordinates (Rule 2), Eq. 21 shows that the proper order goes from right to left. An entirely different interpretation is obtained by going left to right (Rule 1): roll about the $z$ body axis, pitch about the $y$ body axis, and yaw about the $x$ body axis. In practice, either point of view could turn out being easier to visualize for a particular case.

$$
\mathbf{R}_{x y z}(\boldsymbol{\theta})=\mathbf{R}_{z}\left(\theta_{z}\right) \cdot \mathbf{R}_{y}\left(\theta_{y}\right) \cdot \mathbf{R}_{x}\left(\theta_{x}\right)
$$

Euler chose a different set of three sequential rotations to represent an arbitrary orientation. Fig. 14 shows the Euler angles $\phi, \theta$ and $\psi$ taken about the body coordinate axes $z, y^{\prime}$ and $z^{\prime \prime}$. Eq. (22) shows the proper order from left to right: rotate about the $z$ axis, rotate about the $y^{\prime}$ axis, and rotate about the $z^{\prime \prime}$ axis. It differs from the roll-pitch-yaw representation only in the last rotation.

$$
\mathbf{R}_{\text {Euler }}(\phi, \theta, \psi)=\mathbf{R}_{z}(\phi) \cdot R_{y^{\prime}}(\theta) \cdot \mathbf{R}_{z^{\prime \prime}}(\psi)
$$

A third way to describe an arbitrary orientation is with a single angle rotation about a fixed axis. The vector direction of the axis and the magnitude of the rotation can be expressed as an angle vector in the base CS. This transformation equivalently represents simultaneous rotations about the base $x, y$ and $z$ axes. It may be constructed from a sequence of rotations as follows. The first two rotations establish a temporary CS that has its $z$-axis aligned to the angle vector $\boldsymbol{\theta}$. Once the desired rotation is computed in the temporary CS, it can be transformed back to the base CS as $\mathbf{R}_{\text {temp }} \mathbf{R}_{\mathrm{z}}(\|\boldsymbol{\theta}\|) \mathbf{R}_{\text {temp }}{ }^{\mathrm{T}}$. An algebraic expansion of this sequence eventually simplifies to a manageable result given in Eq. (23).

$$
\begin{aligned}
\mathbf{R}_{\text {axis }}(\boldsymbol{\theta})= & \cos (\|\boldsymbol{\theta}\|)\left[\begin{array}{ccc}
1 & 0 & 0 \\
0 & 1 & 0 \\
0 & 0 & 1
\end{array}\right] \\
& +\frac{\sin (\|\boldsymbol{\theta}\|)}{\|\boldsymbol{\theta}\|}\left[\begin{array}{ccc}
0 & -\theta_{z} & \theta_{y} \\
\theta_{z} & 0 & -\theta_{x} \\
-\theta_{y} & \theta_{x} & 0
\end{array}\right] \\
& +\frac{1-\cos (\|\boldsymbol{\theta}\|)}{\|\boldsymbol{\theta}\|^{2}}\left[\begin{array}{c}
\theta_{x} \\
\theta_{y} \\
\theta_{z}
\end{array}\right] \cdot\left[\theta_{x} \theta_{y} \theta_{z}\right]
\end{aligned}
$$

The elementary matrix that accounts for the translation between CS's is the cross product matrix, which performs the familiar vector cross product operation as a matrixvector multiplication. In the context of Mechanics, the cross product relates through a lever arm either a force to a moment or an infinitesimal rotation to an infinitesimal translation. A point to notice is that each process must go in the direction stated; that is, the inverse process is ambiguous. For example, there are infinitely many forces that can act on the same lever arm to give the same moment. In addition, the order that the vectors appear in the cross product is important. Eq. (24) gives the order for forces and moments and Eq. (25) gives the order for infinitesimal rotations and translations. The non-invertible cross product matrix properly expresses the unidirectionality of the three-dimensional transformation involving only the lever arm. When extended to six dimensions, the transformation becomes invertible.

$$
\begin{aligned}
& {\left[\begin{array}{l}
m_{x} \\
m_{y} \\
m_{z}
\end{array}\right]=} {\left[\begin{array}{l}
r_{x} \\
r_{y} \\
r_{z}
\end{array}\right] \times\left[\begin{array}{l}
f_{x} \\
f_{y} \\
f_{z}
\end{array}\right] } \\
&= {\left[\begin{array}{lll}
0 & -r_{z} & r_{y} \\
r_{z} & 0 & -r_{x} \\
-r_{y} & r_{x} & 0
\end{array}\right] \cdot\left[\begin{array}{l}
f_{x} \\
f_{y} \\
f_{z}
\end{array}\right] } \\
& \mathbf{m}=\mathbf{r} \times \mathbf{f}=\mathbf{C}(\mathbf{r}) \cdot \mathbf{f} \\
& {\left[\begin{array}{l}
d \delta_{x} \\
d \delta_{y} \\
d \delta_{z}
\end{array}\right]=\left[\begin{array}{c}
d \theta_{x} \\
d \theta_{y} \\
d \theta_{z}
\end{array}\right] \times\left[\begin{array}{c}
r_{x} \\
r_{y} \\
r_{z}
\end{array}\right] } \\
&=\left[\begin{array}{ccc}
0 & r_{z} & -r_{y} \\
-r_{z} & 0 & r_{x} \\
r_{y} & -r_{x} & 0
\end{array}\right] \cdot\left[\begin{array}{l}
d \theta_{x} \\
d \theta_{y} \\
d \theta_{z}
\end{array}\right] \\
& d \boldsymbol{\delta}=d \boldsymbol{\theta} \times \mathbf{r}=\left(d \boldsymbol{\theta}^{\mathbf{T}} \cdot \mathbf{C}(\mathbf{r})\right)^{T}=\mathbf{C}(\mathbf{r})^{\mathrm{T}} \cdot d \boldsymbol{\theta}
\end{aligned}
$$

Eqs. (26) and (27) show, respectively, the previously discussed equilibrium and compatibility relationships expressed as six-dimensional matrix equations. In this expanded form, the equations show separately the cross product matrix and the rotation matrix now extended to six dimensions. Taking Eq. (26) as the defining form, the right-hand matrix transforms the load vector to a rotated CS parallel to $\mathrm{CS}_{0}$ but with the same origin as $\mathrm{CS}_{1}$. Then 
the left-hand matrix completes the transformation by accounting for the offset $\mathbf{r}_{0}$ from $\mathrm{CS}_{0}$ to $\mathrm{CS}_{1}$.

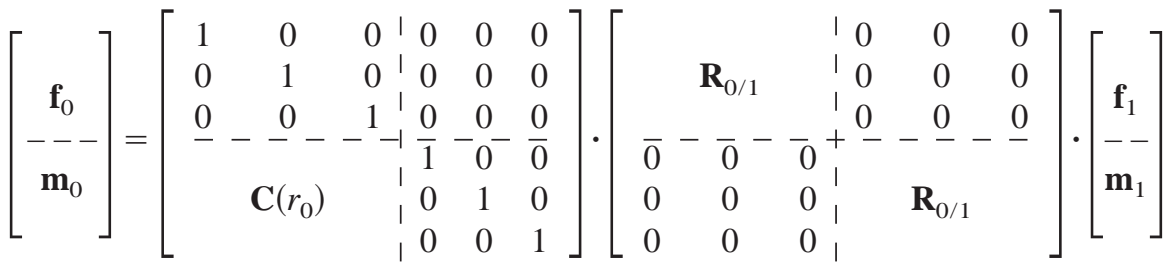

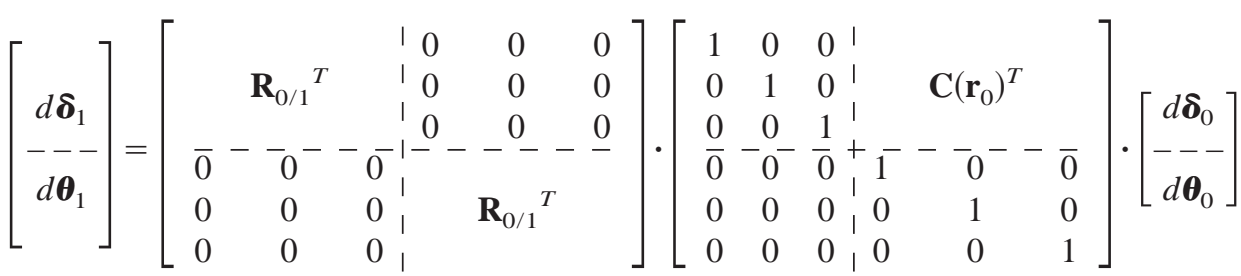

$$
\begin{aligned}
& \mathbf{T}_{0 / 1}=\left[\begin{array}{c:c}
\mathbf{R}_{0 / 1} & \mathbf{0} \\
\hdashline \mathbf{C}\left(\overline{\mathbf{r}}_{0}\right) \cdot \mathbf{R}_{0 / 1}^{-} & \overline{\mathbf{R}}_{0 / 1}^{-}
\end{array}\right]=\left[\begin{array}{c:c}
\mathbf{R}_{1 / 0}^{\mathrm{T}} & \mathbf{0} \\
\hdashline \mathbf{C}\left(\mathbf{r}_{1}^{-}\right) \cdot \mathbf{R}_{1 / 0}^{-} & \mathbf{R}_{1 / 0}^{-}
\end{array}\right] \\
& \mathbf{T}_{1 / 0}=\left[\begin{array}{c:c}
\mathbf{R}_{1 / 0} & \mathbf{0} \\
\hdashline \mathbf{C}\left(\overline{\mathbf{r}}_{1}\right) \cdot \mathbf{R}_{1 / 0}^{-} & \overline{\mathbf{R}}_{1 / 0}^{-}
\end{array}\right]=\left[\begin{array}{c:c}
\mathbf{R}_{0 / 1}^{\mathrm{T}} & \mathbf{0} \\
\hdashline\left(\mathbf{C}^{(}\left(\mathbf{r}_{0}^{-}\right) \cdot \overline{\mathbf{R}}_{0 / 1}\right)^{\mathrm{T}} & \mathbf{R}_{1 / 0}^{-} \mathrm{T}
\end{array}\right]
\end{aligned}
$$

After multiplying the cross product matrix and the rotation matrix, Eq. (28) shows two forms of $\mathbf{T}_{0 / 1}$, the first expressed in $\mathrm{CS}_{0}$ and the second in $\mathrm{CS}_{1}$. Eq. (29) gives the inverse transformation obtained simply by reversing the subscripts. The inverse is easy to prove by multiplying it by the original to get the identity matrix. Notice that either transformation matrix may be inverted simply by transposing the submatrices, although more frequently the first form of Eq. (28) is numerically inverted.

\section{References}

[1] Maxwell, J. C. General Considerations Concerning Scientific Apparatus in The Scientific Papers of James Clerk Maxwell, ed. W. D. Nivens, Dover Press, 1890.

[2] Evans, Chris J. Precision Engineering: An Evolutionary View, Cranfield, England: Cranfield Press, 1989.

[3] Thompson, W, Tait, P. G. Treatise on Natural Philosophy, Cambridge, 1879,. Reprinted as Principles of Mechanics and Dynamics, Dover Publications, New York, 1912:1962.

[4] Slocum, Alexander H. Kinematic couplings for precision fixturing - Part I: Formulation of design parameters Prec Eng, 1988, 10(2), 85-91.

[5] Slocum, A. H, and Donmez, A. Kinematic couplings for precision fixturing - Part II: Experimental determination of repeatability and stiffness Prec Eng, 1988, 10(3), 115-121.

[6] Poovey, Holms, Trumper,. A kinematically-coupled magnetic bearing calibration fixture Prec Eng, 1994, 16(2), 99-108.

[7] Johnson, K. L. Contact Mechanics, Cambridge University Press,., 1985.

[8] Slocum, Alexander H. Design of three-groove kinematic couplings Prec Eng, 1992, 14(2), 67-76.

[9] Slocum, Alexander H. Precision Machine Design, Englewood Cliffs, NJ. Prentice Hall, 1992.

[10] Schmiechen, P, Slocum, A. Analysis of Kinematic Systems: A Generalized Approach Prec Eng, 1996, 19(1), 11-18.

[11] Blanding, Douglass L. Exact Constraint: Machine Design Using Kinematic Principles, ASME Press, New York, 1999.

[12] Schouten, Rosielle, Schellekens Design of a kinematic coupling for precision applications Prec Eng, 1997, 20(1), 46-52.

[13] Hale, Layton C. Friction-Based Design of Kinematic Couplings Proc ASPE,.. 18, 45-48, (UCRL-JC-131297), 1998.

[14] Hale, Layton C. Principles, and Techniques for Designing Precision Machines,., Ph.D. Thesis, M.I.T. Cambridge Massachusetts, (UCRLLR-133066), 1999. Note: UCRL documents are available on line at http://www.llnl.gov/.

[15] Paul, Richard P. Robot Manipulators, The MIT Press, Cambridge, MA, 1981.

[16] Strang, Gilbert Introduction to Applied Mathematics, WellesleyCambridge Press, Wellesley, MA, 1986.

[17] Hale, Layton C. Three-Tooth Kinematic Coupling, U.S. Patent 6,065,898, May 23, 2000 